\title{
Krankheit und Sinn: Zur religiösen Wahrnehmung von Krankheit und ihren ethischen Implikationen
}

Fischer, Johannes

\begin{abstract}
Der Beitrag befasst sich mit religiösen Deutungen von Krankheit und ihren ethischen Implikationen, und zwar aus einer christlichen Perspektive. Er problematisiert die verbreitete Auffassung, dass Religion es ermöglicht, Kontingenzerfahrungen wie eine Krankheit mit einem Sinn zu verbinden und dadurch in das eigene Leben zu integrieren. Das Spezifische religiöser Deutungen und Praktiken liegt eher darin, dass sie es ermöglichen, mit Sinnlosigkeit zu leben. Sie haben dabei ethische Implikationen in Bezug auf den Umgang mit Krankheit und Sterben.
\end{abstract}

DOI: https://doi.org/10.1007/s00481-010-0118-4

Other titles: Disease and its meaning: on the religious perception of disease and its ethical implications

Posted at the Zurich Open Repository and Archive, University of Zurich

ZORA URL: https://doi.org/10.5167/uzh-47215

Journal Article

Published Version

Originally published at:

Fischer, Johannes (2011). Krankheit und Sinn: Zur religiösen Wahrnehmung von Krankheit und ihren ethischen Implikationen. Ethik in der Medizin, 23(1):53-61.

DOI: https://doi.org/10.1007/s00481-010-0118-4 


\title{
Krankheit und Sinn: Zur religiösen Wahrnehmung von Krankheit und ihren ethischen Implikationen
}

\author{
Johannes Fischer
}

Online publiziert: 12. Februar 2011

(C) W. Kohlhammer GmbH 2010. Mit freundlicher Genehmigung Springer-Verlag 2011

Zusammenfassung Der Beitrag befasst sich mit religiösen Deutungen von Krankheit und ihren ethischen Implikationen, und zwar aus einer christlichen Perspektive. Er problematisiert die verbreitete Auffassung, dass Religion es ermöglicht, Kontingenzerfahrungen wie eine Krankheit mit einem Sinn zu verbinden und dadurch in das eigene Leben zu integrieren. Das Spezifische religiöser Deutungen und Praktiken liegt eher darin, dass sie es ermöglichen, mit Sinnlosigkeit zu leben. Sie haben dabei ethische Implikationen in Bezug auf den Umgang mit Krankheit und Sterben.

Schlüsselwörter Krankheit $\cdot$ Religion $\cdot$ Sinn $\cdot$ Ethik

\section{Disease and its meaning: on the religious perception of disease and its ethical implications}

\begin{abstract}
Definition of the problem The article is about religious interpretations of illness and their ethical implications, as viewed from a Christian perspective. Arguments The widespread tendency to interpret religion as providing meaning to experiences of contingency (e.g., diseases) and thereby integrating them into one's life is mistaken. Conclusions Religious interpretations and practices should be interpreted as ways of coping with meaninglessness. Their ethical significance is shown with regard to issues concerning illness and dying.
\end{abstract}

Keywords Disease $\cdot$ Religion $\cdot$ Meaning $\cdot$ Ethics

Dieser Beitrag basiert auf einem Vortrag bei der Jahrestagung 2009 „Menschenbilder in der (Medizin-)Ethik“ der Akademie für Ethik in der Medizin (AEM) am 24.-26. September 2009 in Berlin und wurde bereits publiziert in: Fischer, Johannes (2010) Krankheit und Sinn: Zur religiösen Wahrnehmung von Krankheit und ihren ethischen Implikationen. In: Fischer, Johannes. Sittlichkeit und Rationalität. W. Kohlhammer GmbH, Stuttgart, S 318-327.

J. Fischer $(\bowtie)$

Institut für Sozialethik, Ethik-Zentrum der Universität Zürich, Zollikerstr. 117, 8008 Zürich, Schweiz

E-Mail: fischer@sozethik.uzh.ch 
I

Das Thema dieser Tagung sind Menschenbilder in der Ethik und speziell in der Medizinethik. Mein Vortrag fällt hier etwas aus dem Rahmen, und so will ich eine Bemerkung vorausschicken zu dessen Einordnung in das Gesamtthema der Tagung. Es geht hier nicht um das Verständnis des Menschen, das der Medizin, der Medizinethik oder der Ethik insgesamt zugrunde liegt, sondern vielmehr darum, wie ein kranker Mensch sich selbst und seine Situation versteht oder verstehen kann, also um mögliche Deutungen von Krankheit, und zwar in religiöser Perspektive. Auch dabei geht es in einem weiten Sinne um das Verständnis des Menschen oder, wenn man es so nennen will, um „Menschenbilder“, die sich positiv, aber auch negativ auswirken können auf den Umgang mit Krankheit und deren existenzielle Bewältigung.

Der Beitrag hat drei Teile. Der erste befasst sich mit der Frage, was man sich unter einer religiösen Wahrnehmung von Krankheit vorzustellen hat. Ich werde mich dabei auf die christliche Religion beschränken, da ich christlicher Theologe und kein Religionswissenschaftler bin. Im zweiten Teil befasse ich mich mit der Frage nach dem Verhältnis von religiöser Wahrnehmung und Sinn. Mich interessiert dabei die Frage, ob das Charakteristische der religiösen Wahrnehmung von Krankheit tatsächlich darin liegt, dass sie mit dieser einen Sinn zu verbinden erlaubt. Das ist eine verbreitete Vorstellung. Das Spezifische der Religion liegt hiernach darin, dass sie die Kontingenzen und Abgründigkeiten des Lebens mit Sinndeutungen versieht, die es ermöglichen, sie in den Lebenszusammenhang zu integrieren. Ich möchte diese Auffassung problematisieren. Zwar ist nicht zu bestreiten, dass man derartige Formen von Religiosität antrifft, und zwar auch im christlichen Bereich. Doch scheint mir in der jüdisch-christlichen Überlieferung ein anderes Verständnis vorherrschend zu sein. Danach charakterisiert es den religiösen Glauben, dass er es ermöglicht, mit Sinnlosigkeit leben zu können. Die Vorstellung, alles müsse einen Sinn haben, die auf den ersten Blick etwas Hilfreiches und Lebensdienliches zu haben scheint, hat doch bei genauerem Zusehen etwas höchst Ambivalentes, ja Diabolisches, weil sie dazu nötigt oder doch zumindest dazu verleiten kann, selbst noch die größten Abgründigkeiten mit Sinn auszustatten. Im dritten Teil des Beitrags möchte ich auf die ethischen Implikationen der religiösen Wahrnehmung von Krankheit zu sprechen kommen. Ich möchte dabei verdeutlichen, dass diese Wahrnehmung in der Tat Implikationen für die Beurteilung moralischer Fragen haben kann, zum Beispiel im Blick auf die Sterbehilfeproblematik.

Zunächst also zu der Frage, was man sich unter einer religiösen Wahrnehmung von Krankheit vorzustellen hat. Man muss sich hier vergegenwärtigen, dass die religiöse Wahrnehmung einer Krankheit etwas anderes ist als die wissenschaftlich-theologische Reflexion von Krankheit oder Behinderung. Im einen Fall geht es um das Verständnis der Krankheit, das ein Kranker selbst oder sein Umfeld von einer Krankheit hat. Das ist die Perspektive, mit der Mediziner, Pflegende oder Seelsorger konfrontiert sind. Im anderen Fall geht es um die Reflexion auf mögliche Deutungen von Krankheit und ihre innere Stimmigkeit, aber auch ihre Probleme und Aporien im Horizont einer bestimmten religiösen bzw. theologischen Überlieferung. Ich werde auch auf die theologische Dimension zu sprechen kommen. Aber zunächst scheint es mir wichtig, von der konkreten Erfahrung auszugehen, um zu verdeutlichen, wovon wir sprechen, wenn wir von religiöser Wahrnehmung von Krankheit sprechen. Ich beginne daher mit einem Beispiel aus der Seelsorge. Ich war einige Jahre Gemeinde- 
pfarrer, und ich habe damals eine Frau begleitet, die an Krebs erkrankt war und nach der Diagnose noch etwa ein Jahr gelebt hat. In dieser Zeit ist ihr der 31. Psalm zu einem wichtigen Text geworden, den sie sich dann auch für ihre Beerdigung als Predigttext gewünscht hat. Dieser Psalm hat es nicht speziell mit Krankheit zu tun. In ihm adressiert ein Mensch seine Not an Gott, und zugleich gibt er seinem Vertrauen Ausdruck, dass Gott ihn erretten wird. Ich will einige Verse aus diesem Psalm zitieren, und man mag sie sich in der Vorstellung mit der Situation dieser krebskranken Frau verbinden:

Herr, auf dich traue ich, lass mich nimmermehr zuschanden werden, errette mich durch deine Gerechtigkeit!

In deine Hände befehle ich meinen Geist; du hast mich erlöst, Herr, du treuer Gott. Ich freue mich und bin fröhlich über deine Güte, dass du mein Elend ansiehst und nimmst dich meiner an in Not und übergibst mich nicht in die Hände des Feindes; du stellst meine Füße auf weiten Raum.

Herr, sei mir gnädig, denn mir ist angst! Mein Auge ist trübe geworden vor Gram, matt meine Seele und mein Leib.

Denn mein Leben ist hingeschwunden in Kummer, und meine Jahre mit Seufzen.

Vor allen meinen Bedrängern bin ich ein Spott geworden, eine Last meinen Nachbarn und ein Schrecken meinen Bekannten.

Die mich sehen auf der Gasse, fliehen vor mir. Ich bin vergessen in ihren Herzen wie ein Toter, ich bin geworden wie ein zerbrochenes Gefäß.

Ich aber, Herr, hoffe auf dich und spreche: Du bist mein Gott! Meine Zeit steht in deinen Händen ([4], Psalm 31,2.6.8-13.15 f.).

Wenn man sich die Situation der Frau vergegenwärtigt, die dieser Text in ihrer Krankheit begleitet hat, dann wird hieran etwas deutlich von dem, was man als ,religiöse Wahrnehmung von Krankheit" bezeichnen kann. Der Text fungiert als eine Art Medium, das es der Frau ermöglicht, die Krankheit mit allem, was sie an Leid, Angst und Verzweiflung bedeutet, vor Gott zu bringen und damit in ein bestimmtes Bezugssystem zu stellen, das durch diesen Psalm aufgespannt wird. Ich habe dieses Beispiel gewählt im Blick auf die gleich zu erörternde Frage nach dem Verhältnis von religiöser Wahrnehmung und Sinn. In Psalm 31 wird die Not, von der die Rede ist, an keiner Stelle mit Sinndeutungen in Verbindung gebracht. Sie bleibt vielmehr in ihrer Abgründigkeit stehen. Das wirft die Frage auf, worin das Tröstliche dieses Textes liegt, das er offenbar für diese Frau gehabt hat. Einmal sicherlich darin, dass er deren Krankheitserfahrung in ein Bezugssystem stellt, das es ermöglicht, trotz allem am Vertrauen festzuhalten. Doch scheint mir in diesem Zusammenhang noch etwas anderes wichtig zu sein, nämlich dass der Psalm eine Sprache zur Verfügung stellt, die es ermöglicht, diese Erfahrung überhaupt zu artikulieren und damit eine Distanz zu gewinnen von der unmittelbaren Überflutung durch Angst und Verzweiflung.

Ich will das Gemeinte noch an einem anderen Beispiel illustrieren. Der Neurologe Oliver Sacks schildert in seinem Buch „Der Tag, an dem mein Bein fortging“, wie er sich bei einer Wanderung in der Einsamkeit der Berge Norwegens ein Bein bricht. Auf einen Schlag wird die Einsamkeit, die er eben noch genossen hat, zur tödlichen Bedrohung. Sacks schildert, was ihm bei seinem Überlebenskampf durch den Kopf ging, Bilder, Texte, Musik, und auch Verse aus dem Buch des Predigers, seit seiner Jugend nicht mehr gelesen, ,ein jegliches hat seine Zeit, und alles Vorhaben unter dem Himmel hat seine Stunde; geboren werden hat seine Zeit, und Sterben hat seine Zeit“ ([4], Prediger 3,1 f.), diese, wie Sacks schreibt, „seltsame, tiefe, ruhige Klarheit, die weder kalt noch heiß, weder streng noch nachgiebig, sondern überaus wunderbar, schrecklich aufrichtig war" ([5], S.22). 
Es wird in dieser Schilderung deutlich, was religiöse und in gewissem Sinne auch literarische Texte ermöglichen: Ein Sich-Einfinden mit seinem Erleben in Texte, die diesem Erleben Ausdruck verleihen und die eben damit, dass sie dies tun, von dem unmittelbaren, diffusen, Panik und Angst auslösenden Erlebensdruck befreien und in die Distanz eines artikulierten Umgangs mit der eigenen Erfahrung versetzen, in der sich diese ,seltsame, tiefe, ruhige Klarheit“ einstellen kann. Dieses Versetztwerden in die Distanz zur eigenen Erfahrung ist selbst eine Erfahrung, die durch derartige Texte, aber auch durch religiöse Praktiken und Riten ermöglicht wird, und wenn man dies noch einmal auf Psalm 31 zurück bezieht, dann kann sich einem Menschen in dieser Erfahrung etwas von der Errettung mitteilen, um die der Psalm fleht, auch wenn die Not, die der Grund für das Flehen ist, nicht verschwunden ist.

Dies ist ein wichtiger Aspekt religiöser Erfahrung, der hervorzuheben ist gegenüber einer Auffassung, wonach sich die religiöse Erfahrung durch ihren besonderen Gegenstand, nämlich das Heilige oder die Transzendenz, von anderen Arten der Erfahrung unterscheidet, wobei dieser Gegenstand als etwas gedacht wird, das der sprachlichen Artikulation in den verschiedenen religiösen Semantiken voraus liegt. Die religiöse Erfahrung wird damit zu etwas Exzeptionellem, das mit einem überwältigenden Gefühl von Erhabenheit verbunden ist. In dieser Auffassung liegt die Gefahr, dass die gelebte Religiosität entwertet wird, da in ihr solche exzeptionellen Erfahrungen eher selten sind. Wie die Beispiele verdeutlichen, sind es eher umgekehrt die sprachlichen Artikulationen und Praktiken einer Religion, die religiöse Erfahrungen freisetzen, indem sie das eigene Leben in ein bestimmtes Bezugssystem stellen.

III

In Bezug auf das Verhältnis von religiöser Wahrnehmung und Sinn befasst möchte ich hier zunächst eine Unterscheidung einführen, nämlich zwischen der Bedeutung und dem Sinn einer Krankheit. Wenn ich im Folgenden von Bedeutung spreche, dann ist damit dasjenige gemeint, was durch Fragen artikuliert wird wie: „Was bedeutet es für einen Menschen zu erfahren, dass er aufgrund einer terminalen Erkrankung nicht mehr lange zu leben hat?", oder: „Was bedeutet es für einen Menschen, unter Bedingungen extremer Armut leben zu müssen?" Solche Fragen fordern das Verstehen heraus, und wir verstehen die Bedeutung von etwas, indem wir uns die betreffende Situation oder Lebenslage vor Augen führen. Man stelle sich einen Menschen vor, der soeben die Diagnose einer terminalen Krankheit erfahren hat und nun aus der Klinik hinaus auf die Straße tritt. Er findet dort alles so vor wie noch eine Stunde zuvor: Die Sonne scheint, die Bäume stehen im Grün des Sommers, die Leute gehen inmitten des pulsierenden Verkehrs ihren Geschäften nach, in den Anlagen spielen die Kinder. Alles ist wie zuvor - und dennoch ist nichts mehr wie zuvor. Denn dazwischen ist das Gefühl getreten, dass dies das Leben der anderen ist und dass das eigene Leben plötzlich seinen Ort darin verloren hat. Die Bedeutung einer schweren Erkrankung manifestiert sich in derartigen Veränderungen des Wahrnehmens, des Fühlens und Denkens oder, wie man auch sagen kann, in derartigen existenziellen Erschütterungen, denen der Kranke, aber auch seine Angehörigen ausgesetzt sind. Diesbezüglich macht es keinen Unterschied, ob ein Mensch religiös ist oder nicht. Auch die Frau in dem Beispiel, von dem ich ausgegangen bin, hat ihre Krankheit in dieser Weise erlebt, und die Bibel - man denke an Hiob - ist voll von Beispielen dieser Art.

Auch wenn wir von Sinn sprechen, geht es um Verstehen. Der Unterschied zum Verstehen der Bedeutung, die etwas hat, besteht darin, dass die Frage nach dem Sinn von etwas 
mit einer Unterstellung verbunden ist, nämlich dass das Betreffende in einer Beziehung zu etwas anderem steht, aus der es verständlich wird. Gemeint ist nicht eine Kausalbeziehung, also z. B. die Rückführung einer Krankheit auf bestimmte Ursachen. Eine Kausalerklärung bezieht sich auf die Krankheit als ein physisches oder psychisches Ereignis. Bei der Frage nach dem Sinn einer Krankheit geht es demgegenüber darum, die Krankheit zu etwas in Beziehung zu setzen, aus dem verständlich wird, warum sie gerade mir als dieser Person zustößt. Die Frage nach dem Sinn einer Krankheit ist eine Reaktion auf das, was ich die Bedeutung einer Krankheit nenne, nämlich auf die existenzielle Erschütterung, die mit der Erkrankung verbunden ist und die die Person des Kranken aus dem vertrauten Lebenszusammenhang herausfallen lässt. Sie zielt darauf ab, im Gegenzug die Krankheit wieder in den Lebenszusammenhang einzuordnen, indem sie sie aus diesem heraus verständlich zu machen sucht. Dies geschieht über bestimmte Muster, die unsere religiöse und kulturelle Überlieferung ausgebildet hat. Ein Beispiel ist das Muster des Tun-Ergehen-Zusammenhangs, dem die Warum-Frage zugrunde liegt: Warum stößt die Krankheit gerade dieser Person zu? Der Kranke hat falsch gelebt oder etwas Falsches getan, und die Krankheit ist die Folge davon oder die Strafe dafür. Dieses Muster spielt zum Beispiel in der Hiob-Erzählung eine Rolle. Ein anderes Beispiel ist das Muster der Läuterung, das an der Wozu-Frage orientiert ist: Wozu ist die Krankheit dieser Person geschickt? Auch hier hat der Kranke falsch gelebt, zum Beispiel bestimmte seelische Probleme ignoriert oder verdrängt, und die Krankheit hat den Sinn, ihm dies aufzudecken, damit er sein Leben korrigieren kann. Insofern eröffnet sie dem Kranken eine Chance. Wenn ich es richtig sehe, dann spielt dieses Muster in der medizinischen Anthropologie von Viktor von Weizsäcker eine Rolle.

Ich sagte, dass Psalm 31, von dem ich in meinem Eingangsbeispiel ausgegangen bin, sich jeder Sinndeutung enthält. Dagegen mag vielleicht eingewendet werden, dass jener Frau dieser Psalm dazu diente, ihre Krankheit zu Gott in Beziehung zu setzen. Wird die Krankheit hier also nicht doch in einen Sinnzusammenhang eingeordnet? Dies kann nur auf den ersten Blick so erscheinen. Tatsächlich diente dieser Psalm der Frau nicht dazu, eine Beziehung zwischen der Krankheit und Gott herzustellen, sei es im Sinne der Warum- oder der Wozu-Frage, sondern vielmehr dazu, die Krankheit und die Erschütterung, die sie bei ihr auslöst, vor Gott zu bringen. Ob Gott mit der Krankheit etwas zu tun hat, ob sie also in irgendeiner Beziehung zu Gott steht, bleibt dabei offen.

Die Sinnfrage hat etwas Ambivalentes. Es gibt Beispiele, bei denen uns dies sofort evident ist. Was war der Sinn des Holocaust? Jeder Versuch, die Ermordung von Millionen von Menschen mit einem Sinn in Verbindung zu bringen und sie damit in unsere vertraute Sicht der Dinge einzuordnen, hat etwas Abgründiges. Jan Philipp Reemtsma äußerte in einem Vortrag über das Phänomen der Gewalt die Auffassung, dass die Frage, wie ,normale“ - also nicht psychisch abnorme - Menschen so etwas haben tun können, eigentlich ,albern“ sei. Denn die Geschichtsschreibung habe diese Frage längst beantwortet, indem sie die Ereignisse nachgezeichnet hat, die zum Holocaust geführt haben. Doch scheint mir dies nicht den Sinn dieser Frage zu treffen. Sie zielt nicht auf eine historische Erklärung, sondern sie artikuliert das blanke Unverständnis im Hinblick auf die Einordnung des Entsetzlichen in unsere vertraute Sicht der Dinge: Wie konnten Menschen so etwas tun? Die Frage zielt also auf genau das, was das Böse als Böses charakterisiert, nämlich seine Unbegreiflichkeit. Die Frage nach dem Sinn des Holocausts ist ein zwar verständlicher, aber abgründiger Versuch, das schlechthin Unbegreifliche in Verständlichkeit zu überführen und es in unsere vertraute Sicht der Dinge zu integrieren.

Diese Ambivalenz der Sinnfrage lässt sich gerade an unserer religiösen Überlieferung beobachten. Denn das menschliche Bedürfnis, sich das Unverständliche verständlich zu machen und es damit in die vertraute Sicht der Dinge einzuordnen, hat auch in dieser Über- 
lieferung eine Rolle gespielt. Es sind vor allem drei Sinndeutungen gewesen, mit denen man sich das menschliche Leiden begreiflich zu machen suchte, und von zwei von ihnen war bereits die Rede (vgl. dazu [2], S. 45-56). Erstens ist das menschliche Leiden Strafe Gottes für die menschliche Sünde. Zweitens hat Gott das Leiden über den Menschen verhängt, um ihn zu erziehen und zu läutern, seine Geduld auf die Probe zu stellen und seine Tugend zu mehren. Drittens liegt nach dem Willen Gottes im Leiden der Heilsweg für den Menschen. Denn Gott hat den Menschen durch das Kreuz Jesu von der Sünde erlöst, und das legt die Vorstellung nahe, dass der Christ im Leiden sein Kreuz auf sich nehmen und Jesus gleichförmig werden soll, um mit ihm zur ewigen Herrlichkeit zu gelangen.

Es ist hier nicht Raum, diese Sinndeutungen des Leidens einer eingehenden theologischen Kritik zu unterziehen. Das theologisch Fragwürdige liegt vor allem in dem Gottesbild, das sie zur Folge haben: ein Gott, der den Menschen mit Strafe für seine Sünde überzieht; Gott als „Vertreter einer ,schwarzen“ Pädagogik“ ([2], S. 49) im Sinne des Läuterungsgedankens; Gott, der dem Menschen das Leiden als Weg zum Heil verordnet hat. Für die biblische und theologische Tradition konzentriert sich die Problematik der Sinnfrage daher in der Theodizeefrage. Sie lässt sich formulieren als die Frage, wie die Realität menschlichen Leidens vereinbar ist mit drei Eigenschaften, die Gott zugeschrieben werden, nämlich Gottes Allmacht, Gottes Güte und Gottes Verständlichkeit für den Menschen. Wenn Gott allmächtig ist, dann kann er, und wenn er zugleich gütig ist, dann müsste er das menschliche Leiden verhindern. Also stellt die Realität des Leidens Gottes Verständlichkeit für den Menschen in Frage. Jene drei Sinndeutungen des Leidens sind Versuche, dieser Aporie zum Trotz an der Verständlichkeit des Leidens in seiner Beziehung zu Gott festzuhalten. Erkauft ist dies, wie gesagt, mit einem fragwürdigen Gottesverständnis.

Dem korrespondiert auf Seiten des Menschen ein anderes Problem, das die Frage des Widerstands gegen das Leiden betrifft. Wenn im Leiden ein von Gott verfügter Sinn liegt, dann kommt der Widerstand gegen das Leiden einem Aufbegehren gegen Gottes Willen gleich. Im Blick auf eine Krankheit würde dies in der Konsequenz bedeuten, dass der Mensch sich nur in sie ergeben kann. Die Alternative zu dieser Auffassung liegt in dem Gedanken, dass Krankheit an sich selbst etwas Widergöttliches ist und dass es daher gilt, ihr mit allen verfügbaren Mitteln Widerstand entgegenzusetzen, so lange, bis dies keinen Sinn mehr macht und Ergebung die Einstellung ist, die allein noch möglich ist. Unter den evangelischen Theologen hat besonders Karl Barth in seiner Schöpfungslehre diesen widergöttlichen Charakter der Krankheit herausgestellt. Unter Verweis auf die Heilungen und Dämonenaustreibungen Jesu begreift er die Krankheit als „ein Werk und eine Kundgebung des Teufels und der Dämonen“, bzw. als „ein Element und Zeichen der die Schöpfung bedrohenden Chaosmacht“ ([1], S. 417). Und er leitet daraus ab: „Was der Mensch diesem ganzen Reich zur Linken und so auch der Krankheit gegenüber in Übereinstimmung mit dem Willen Gottes wollen soll, kann immer nur Widerstand bis auf das Letzte sein“ ([1], S. 418). So begriffen ist Krankheit das schlechterdings Sinnlose.

Viktor von Weizsäckers medizinische Anthropologie bietet mit ihrer These, dass eine Krankheit einen verborgenen Sinn hat, der im Gespräch zwischen Arzt und Patient entschlüsselt werden soll, in gewisser Weise das säkulare Gegenmodell zu dieser Barthschen Auffassung der Krankheit. Die Krankheit ist hier nicht Einbruch des Chaos in die Ordnung des Lebens, sondern Ausdruck dieser Ordnung, nämlich als Indikator dafür, dass diese Ordnung ins Ungleichgewicht geraten ist und wiederhergestellt werden muss. Darin liegt ihr Sinn. Es ist hier nicht Raum, in diese Diskussion einzutreten (vgl. dazu [3], S. 163-176). Ich will dazu nur so viel anmerken, dass die Barth'sche Auffassung der Krankheit keineswegs ausschließt, dass ein Kranker selbst seine Krankheit so begreifen kann, dass sie ihm einen neuen Blick auf sich selbst, aber auch für die im Leben wesentlichen Dinge erschlossen hat und dass sie 
ihn dazu geführt hat, sein Leben grundlegend zu ändern. Vielleicht kann er solchermaßen mit der Krankheit einen Sinn für sich selbst verbinden. Dies ist zweifellos ein wichtiger Aspekt für die Bewältigung einer Krankheit, und das betrifft nicht nur die Seelsorge, sondern es kann auch die Arzt-Patienten-Beziehung betreffen. Doch muss diese Verarbeitung einer Krankheit durch den Kranken selbst unterschieden werden von der These, dass eine Krankheit stets und als solche einen Sinn hat, den es aufzuspüren und zu verstehen gilt.

Nun wären diese Überlegungen unvollständig, wenn nicht noch der folgende Gedanke hinzukommt. Ich bin ausgegangen von dem Beispiel der an Krebs erkrankten Frau, für die der 31. Psalm zu einem wichtigen Text geworden ist. Wenn der Beter des 31. Psalms seine Not an Gott adressiert, dann tut er dies in der Hoffnung, dass Gott ihn aus seiner Not errettet. Insofern wird hier nun doch eine Beziehung zwischen dieser Not und Gott hergestellt, zwar nicht so, dass diese auf Gott zurückgeführt und von daher in ihrem Sinn verständlich gemacht wird, aber doch im Sinne der Erwartung, dass Gott diese Not wenden kann. Dem entspricht das Vertrauen, es, wie immer die Dinge ausgehen, letztlich mit Gott zu tun zu haben. Dieses Vertrauen findet innerhalb der christlichen Spiritualität seinen Ausdruck in der Vaterunser-Bitte „Dein Wille geschehe“. Die Führung des eigenen Lebens bekommt damit den Charakter eines Sich-Führen-Lassens. Das ist nicht mit Fatalismus zu verwechseln, sondern in dieser Einstellung kann gerade eine Ressource liegen dafür, sich der eingetretenen Situation stellen zu können und Verantwortung zu übernehmen für das, was nun zu entscheiden und zu tun ist. Wenn Religion etwas ermöglicht, dann vor allem dieses: mit Sinnlosigkeit leben zu können. Darin kann eine Befreiung liegen von dem Zwang, alles, und selbst noch das Böse, mit Sinn unterlegen zu müssen, um es in die vertraute Sicht der Dinge zu integrieren.

Worin liegen die ethischen Konsequenzen der religiösen Wahrnehmung von Krankheit? Es ist deutlich geworden, dass es schon innerhalb unserer eigenen religiösen Tradition nicht „die“ religiöse Wahrnehmung von Krankheit gibt, sondern dass Krankheit unterschiedlich wahrgenommen und gedeutet worden ist. Da sind einerseits Sinnkonstruktionen, die die Krankheit auf Gott als ihren Urheber zurückführen und sie solchermaßen verständlich zu machen suchen. Und da ist andererseits die Auffassung der Krankheit als etwas Widergöttliches, als Einbruch der Chaosmächte in das menschliche Leben, wie Karl Barth es begriffen hat.

Was das Erste, nämlich die Sinnkonstruktionen betrifft, so dürfte deutlich sein, dass sie weitreichende ethische Konsequenzen haben können. Wenn z. B. Erbkrankheiten wie eine Chorea Huntington von Gott verfügt sind und von daher einen Sinn haben, dem der Mensch sich nur fügen kann, mag uns dieser Sinn auch letztlich nicht begreifbar sein, dann wird man zu der Auffassung tendieren, dass sich die Selektion von Embryonen bei der PID mit dem Ziel, die Weitervererbung solcher Krankheiten zu verhindern, grundsätzlich verbietet. Die Frage würde eine eigene Diskussion erfordern, ob nicht Vorstellungen dieser Art in der kirchlichen und theologischen Debatte über die PID zumindest hintergründig eine bedeutende Rolle spielen.

Was das Zweite betrifft, nämlich das Verständnis des religiösen Glaubens im Sinne eines Sich-Führen-Lassens inmitten von Sinnlosigkeitserfahrungen, so hat auch dies für diejenigen, die dieses Verständnis teilen, weitreichende ethische Implikationen. Man mag sich dies an der Suizidbeihilfedebatte verdeutlichen. Wer sein Leben in dieser Weise versteht, für den wird sich in Anbetracht einer schweren Erkrankung die Frage stellen, ob ein Suizid eine mögliche Option sein kann oder ob es nicht auch hier um ein Sich-Führen-Lassen geht im 
Sinne des Annehmens der Krankheit mit allem, was sie an Einschränkungen, Leiden und Belastungen für Dritte bedeutet. Das hat Konsequenzen auch für die Frage der Beihilfe zum Suizid. Hier wird sich für ihn die Frage stellen, ob er Beihilfe zu einer Handlung leisten kann, die er aufgrund seiner eigenen Lebensauffassung für keine mögliche Option hält. Man muss es bei diesen Fragen belassen, die diejenigen, welche eine solche Sicht des Lebens teilen, letztlich nur für sich selbst beantworten können.

Schließlich sei noch ein dritter ethischer Aspekt genannt. Ich habe das, was ich die Bedeutung einer Krankheit nenne, als Unterbrechung bzw. Destruktion des vertrauten Lebenszusammenhangs umschrieben. Die Unterstellung, dass die Krankheit einen Sinn haben muss, versucht im Gegenzug die Krankheit in den vertrauten Lebenszusammenhang einzuordnen. Die Alternative hierzu liegt darin, den Kranken in einen neuen Lebenszusammenhang zu integrieren, zu dem seine Krankheit gehört mit allem, was sie für ihn bedeutet, und in diesem Sinne hat die christliche Überlieferung Krankheit und Sterben als etwas begriffen, das zu besonderer Fürsorge und Begleitung verbindet. Zu denken ist hier etwa an Matthäus 25,36, wo Jesus von sich selbst sagt: „Ich bin krank gewesen, und ihr habt mich besucht.“ Das hat innerhalb der christlichen Diakonie das Verständnis der Krankheit geprägt: Im Kranken ist Jesus selbst gegenwärtig, und was dem Kranken getan wird, das wird ihm getan. Die Fürsorge für die Kranken ist von daher ein wesentlicher Bestandteil des christlichen Ethos. Dies ist für das Thema dieser Tagung von Bedeutung, weil es auf den engen Zusammenhang zwischen dem Verständnis von Krankheit und dem zugrunde liegenden Menschenbild verweist. Der Gedanke, dass es angesichts des Zerbrechens des vertrauten Lebenszusammenhangs darum geht, den Kranken erneut in einen Lebenszusammenhang zu integrieren dadurch, dass ihm Begleitung und Fürsorge zugewendet werden, liegt z. B. der Hospizbewegung zugrunde, und in diesem Sinne lässt sich auch die Forderung nach einem Ausbau der Palliativbetreuung verstehen in dem weiten Sinne, in welchem sie auch die seelische Betreuung des Kranken umfasst.

Es mag an diesen Beispielen deutlich werden, dass sich moralische Fragen, gerade wenn sie Krankheit und Sterben betreffen, nicht abtrennen lassen von Fragen des Selbstverständnisses und des Verständnisses der Lebenswirklichkeit im Ganzen. Ich bin mir bewusst, dass dieser Punkt in der heutigen ethischen Debatte kontrovers ist. Nach verbreiteter Auffassung hat es die Moral mit dem Allgemeinen im Sinne des für jedermann Gültigen zu tun, und das schließt ihre Fundierung in partikularen religiösen oder weltanschaulichen Überzeugungen aus. Ich für meinen Teil glaube nicht, dass es eine allgemein-menschliche Moral gibt, die für die Menschen aller Zeiten und Zonen Gültigkeit hat und die lediglich bei noch unaufgeklärten Menschen durch religiöse Überzeugungen überlagert und verdeckt ist. Das, was uns als Angehörigen des westlichen Kulturkreises als Moral gilt, ist das Produkt einer kulturellen Entwicklung, für die das Christentum und insbesondere die Reformation entscheidende Weichen gestellt haben (vgl. dazu [6]). Es ist daher eng mit unserem Selbst- und Wirklichkeitsverständnis verwoben, und im globalen Horizont betrachtet hat es selbst einen partikularen Charakter, was sich etwa an der Schwierigkeit zeigt, sich auf globale bioethische Standards zu verständigen. Solche Erwägungen bedürften freilich einer eigenen Erörterung.

\section{Literatur}

1. Barth K (1957) Kirchliche Dogmatik III/4. Evangelischer Verlag, Zollikon-Zürich

2. Brantschen JB (2004) Vom Umgang mit Schmerz und Leiden - aus christlicher Perspektive. In: Stulz P (Hrsg) Theologie und Medizin. Ein interdisziplinärer Dialog über Schmerz und Leiden, Heil und Heilung. Chronos Verlag, Zürich, S 45-56 
3. Etzelmüller G (2009) Der kranke Mensch als Thema theologischer Anthropologie. Die Herausforderung der Theologie durch die anthropologische Medizin Viktor von Weizsäckers. Z Evang Ethik 3:163-176

4. Evangelische Kirche in Deutschland, Bund der Evangelischen Kirchen in der DDR (Hrsg) (1987) Die Bibel. Nach der Übersetzung Martin Luthers. Deutsche Bibelgesellschaft, Stuttgart

5. Sacks O (1989) Der Tag, an dem mein Bein fortging. Rowohlt, Reinbek bei Hamburg

6. Taylor C (1996) Quellen des Selbst. Die Entstehung der neuzeitlichen Identität. Suhrkamp, Frankfurt a.M. 